\title{
Die Explizierung des Impliziten
}

\section{Kommentar zu Hans Wußing: Zur Entstehungsgeschichte des abstrakten Gruppenbegriffs (1965)}

\author{
Erhard Scholz
}

„Ganz unverkennbar rückt seit einiger Zeit der Begriff ,Mathematische Struktur' zu einem der zentralen Begriffe der modernen Mathematik auf". So beginnt der Beitrag von Hans Wußing (geb. 1927). Mitte der 1960er Jahre befand sich die moderne Mathematik mit ihrer mengen- und strukturorientierten Auffassung auf ihrem Höhepunkt. Durch ihren - nicht sehr glücklichen - Auftritt innerhalb der schulischen Lehre der OECD-Länder in Form einer trivialisierten Form der Mengenlehre begann sie um diese Zeit ihren Weg in eine breitere Öffentlichkeit. Für die Wissenschaftsgeschichte schien es höchste Zeit, sich dieser im 19. Jahrhundert begonnen Bewegung, die sich um die Jahrhundertwende selbst auf den Begriff gebracht hatte (Gray 2009, vgl. auch Mehrtens 1990) in detaillierten historischen Forschungen zuzuwenden. Wußing war einer der Pioniere dieser Arbeitsrichtung innerhalb der Mathematikgeschichte. Der hier besprochene Beitrag ist eine Kurzzusammenfassung der Ergebnisse seiner im April 1965 abgeschlossenen Habilitationsschrift. Sie wurde vier Jahre später unter dem Titel Die Genesis des abstrakten Gruppenbegriffs publiziert (Wußing 1969). Im Vorwort beschrieb er den damaligen Stand der

Der Nachdruck des Originalbeitrags ist in diesem Heft dem Kommentar vorangestellt und online unter dem DOI:10.1007/s00048-010-0024-3 Zu finden. 
historischen Forschung zur modernen Mathematikgeschichte wie folgt:

Während die mathematische Entwicklung früherer Perioden teilweise bis in subtilste Einzelheiten hinein erforscht worden ist [...], steht die Historiographie der modernen mathematischen Entwicklung noch am Anfang - trotz F. Kleins ,Vorlesungen über die Entwicklung der Mathematik im 19. Jahrhundert' und trotz der unter dem Pseudonym N. Bourbaki im Jahre 1960 erschienenen ,Élements d'histoire des mathématiques.' (Ebd.: 5).

Dem in der NTM und in diesem Jubiläumsheft nachgedruckten Beitrag folgten neben dem Buch zwei weitere kurze methodische Artikel in derselben Zeitschrift (Wußing 1967, 1970). Zusammen mit seinem Buch legte die Trilogie in der NTM dar, wie sich Wußing - um es nur scheinbar paradox zu formulieren - eine materialistische Begriffsgeschichte der Mathematik vorstellte. Die Erarbeitung allgemeiner Schlüsselbegriffe der Mathematik durch die Mathematiker des 19. und 20. Jahrhunderts sollte zum Zentrum einer Erschließung der Geschichte der gesamten Disziplin - und gegebenenfalls darüber hinaus der theoretischen Naturwissenschaften - gemacht werden. Dabei setzte sich der Autor von der älteren Ideengeschichte dadurch ab, dass er auf die Suche ging, wie sich die Entstehung mathematischer Strukturbegriffe während der mathematischen Arbeit im Material verschiedener Teilgebiete vorbereitete, der jeweilige Begriff hervortrat und schrittweise ausgebildet wurde, bevor er schließlich als geklärter in die jeweiligen Wissensgebiete erkenntnisbildend hineingetragen werden konnte. Wußing spricht in der erstgenannten Phase von einer impliziten Verwendung des Begriffs in statu nascendi, der dann über Hindernisse hinweg schrittweise expliziert und verallgemeinert wird, bevor es zu seiner allgemeinen (etwa axiomatischen) Formulierung kommt. Dem Gegensatz implizit - explizit ordnete er die Gegenüberstellung von Begriffsextension und -intension bei.

Hinsichtlich Intensität und Quellengenauigkeit beim Studium des Auftretens des Begriffs der Gruppe avant la lettre (im impliziten Stadium) oder in seinen verschieden ausformulierten (expliziten) Fassungen unterschied sich Wußings Methodologie also von bloßer Ideengeschichte. Im hier wieder abgedruckten Artikel wird dies in der Beschreibung der drei Wurzeln des Gruppenbegriffs sichtbar. Durch weitere Anreicherungen sollte sie in zweierlei Hinsicht sogar materialistisch werden: Erstens wurde das klassisch-logische Gegensatzpaar Intension/Extension durch die Ostension zu einer Triade der Begriffsdynamik erweitert: „Von lat. se ostendere: sichtbar werden, erscheinen, zu Tage treten “ (Wußing 1970: 21, Anm. 15). Unter ,Ostension' wollte Wußing den gesamten innerwissenschaftlichen wie darüber hinausgehen- 
den gesellschaftlichen Tätigkeitsbereich fassen, ,soweit er für die zu untersuchende Begriffsgeschichte relevant ist" (ebd.: 21). Gingen in diese Wortschöpfung vielleicht noch Anregungen aus der Zeit ein, in der unser Autor als Doktorand der Mathematik Ernst Bloch in naturwissenschaftlichen Fragen zuarbeitete, bevor letzterer Leipzig verlassen musste?

Zweitens wurde von Wußing die Frage gestellt, inwieweit man in der Entwicklung der Mathematik von einer „Äquivalenz des Logischen mit dem Historischen“ ausgehen könne (1967: 24). Er ergänzte sie um den Hinweis auf einen ,,allgemeinen Hintergrund der seit der industriellen Revolution des ausgehenden 18. Jahrhunderts anhaltenden Entwicklung der Produktivkräfte in den kapitalistischen Staaten Europas und Nordamerikas" für die Entwicklung der Mathematik im Allgemeinen und die Herausbildung eines abstrakten Bewegungsbegriffs im Besonderen (1969: 144).

Der letzte Punkt stellte für eine Geschichte der Mathematik aus der Perspektive ihrer grundlegenden Strukturbegriffe eine ungeheure Anforderung dar, wollte man das vielfältige vermittelte Beziehungsnetz zwischen ökonomiegeschichtlichem Hintergrund und begriffshistorischem, Vordergrund' in den Blick bekommen. Auch heute ist dieser große Bogen seitens der Mathematikgeschichte bestenfalls nur in kleinen Ausschnitten beleuchtet. Eine „Äquivalenz des Logischen mit dem Historischen“ wurde innerhalb der Mathematik etwa durch Autoren der genetischen Methode aufgeworfen und von anderen gleich wieder bestritten (Toeplitz 1949, Schubring 1978). Für einige Zeit wurde dieses Thema auch in der Philosophie der Wissenschaften und dem an Marx und Hegel geschulten Teil der Sozialwissenschaften umstritten diskutiert. Während die offizielle Wissenschaftsphilosophie der sowjetisch orientierten staatssozialistischen Länder dazu neigte, eine solche Äquivalenz zu behaupten (auch um eine vermeintliche Notwendigkeit der historischen Entwicklung zum Sozialismus begründen zu können), machte Wußing aus dem Postulat der Äquivalenz eine Frage. Für ihn war es klar, dass die Geschichtsforschung solche Fragen zu präzisieren und aus ihren Studien je einzeln zu beantworten hatte. Wußing war bereit, die Wissenschafts- und Mathematikgeschichte in einen spannungsreichen Dialog mit der Wissenschaftsphilosophie (unterschiedlicher Provenienz) und der allgemeinen Geschichtswissenschaft zu führen.

Der erste Punkt der Erweiterung des Methodenarsenals, die Einbeziehung der ,Ostension' des mathematischen Wissens zielte in eine andere Richtung, auch wenn der Ansatz eher eine Skizze blieb. Die anderthalb wissenschaftliche Generationen später - unabhän- 
gig von Wußings Arbeiten - erfolgende Hinwendung zum Studium epistemischer Praktiken der Wissenschaften sowie der zugehörigen epistemischen Objekte und Systeme (vgl. etwa Rheinberger 1997 für die Molekularbiologie und Epple 1999 für die Mathematik) lässt sich meines Erachtens auch im Sinne der Wußing'schen Ostension wissenschaftlicher Begriffe verstehen. Das lag meines Wissens nicht in der Absicht der Autoren, denn ihre Überlegungen speisten sich aus anderen Quellen. Aber auch in diesen Arbeiten wurde an Detailstudien eine fein ausgearbeitete Methodologie für die historische Untersuchung der praktischen Felder entwickelt, in denen wissenschaftliches Wissen zu Tage tritt.

Wußings Vorstoß in das neue Terrain der Geschichte der jüngeren Mathematik machte Schule und regte weitere Studien an. Am Leipziger Karl-Sudhoff-Institut, an dem Wußings Habilitationsschrift entstand, folgten thematisch ähnlich ausgerichtete Untersuchungen zur Geschichte des Körperbegriffs (Purkert 1971, 1972, 1973) und zur Algebrentheorie (Schlote 1983, 1986/87). Wußing wurde zum Kopf der Leipziger Schule der Mathematikgeschichte und Leiter der Abteilung für die Geschichte der Naturwissenschaften des KarlSudhoff-Instituts sowie wichtiger Vertreter der Wissenschaftsgeschichte in der DDR. Seine Initiative war entscheidend für die Verankerung der Wissenschafts- und speziell der Mathematikgeschichte in der Lehrerausbildung seines Landes. Damit entstand auch eine lebendige Kooperation zwischen Mathematik und Mathematikgeschichte an den Hochschulen der DDR.

Trotz der Reise- und Kontakthindernisse, die aus der damaligen Nicht-Beziehung zwischen den beiden deutschen Staaten resultierten, zeigten Wußings Arbeiten auch in Westdeutschland Wirkung. An der jüngeren Geschichte ihrer Disziplin interessierte Mathematiker lasen sein Buch mit intellektuellem Gewinn. Auch wurde es Anfang der 1970er Jahre in selbstorganisierten studentischen Arbeitskreisen zur Mathematikgeschichte zusammen mit den Büchern von Struik, Klein, Bernal und anderen diskutiert. Mindestens eine mathematikhistorische Dissertation in Westdeutschland wurde in Themenstellung und ansatzweise auch Methode von Wußings Studien zum Gruppenbegriff angeregt (Scholz 1980). Mehrtens (1979) griff ein affines Thema auf.

Auch international stießen die Leipziger Arbeiten auf Interesse und Anerkennung. 1984 erschien Wußings Buch in englischer Übersetzung. In Kooperation mit Erwin Hiebert und anderen bereitete er die Herausgabe einer blockübergreifenden Monographienserie mit dem Titel Science Networks - Historical Studies zur Wissenschaftsgeschichte vor. Sie sollte ein internationales Editorial 
Board haben, in der DDR produziert und in Kooperation zwischen dem VEB Deutscher Verlag der Wissenschaften in Berlin (DDR) und dem Birkhäuser-Verlag in Basel (Schweiz) jeweils in „Ost“ und „West" vertrieben werden. Dieses Vorhaben war meines Wissens ohne Parallele oder gar Vorbild. Die Realisierung erwies sich jedoch als so schwierig, dass die Produktion des ersten Bandes erst in die Wendezeit der DDR fiel. Birkhäuser übernahm den Vertrieb dann ab 1990 allein und führte die Serie unter den veränderten Bedingungen weiter. Die Situation in Leipzig selbst entwickelte sich weniger befriedigend. Nach der Integration der Universität Leipzig in das bundesdeutsche Hochschulsystem wurde die wissenschaftshistorische Abteilung des Karl-Sudhoff-Instituts schrittweise verkleinert und schließlich ganz abgewickelt. Lediglich die medizinhistorische Abteilung überlebte - zumindest bislang.

Mit der Forschungsausrichtung der Leipziger Mathematikgeschichte hatte das nichts zu tun. Das von Hans Wußing erschlossene Themenfeld der Herausbildung der algebraischen Strukturbegriffe erwies sich als fruchtbar und auch international weitertragend (von den anderen Arbeitsschwerpunkten des Leipziger Instituts wäre an anderer Stelle zu berichten). Es wurde zwei (wissenschaftliche) Generationen später in größerer Breite und aus anderer methodischer Perspektive von Leo Corry (1996) neu gesichtet.

Kommen wir daher noch einmal auf die Frage nach der Verträglichkeit der beiden von Wußing geschätzten Aspekte des Impliziten und der Ostension zurück. Entgegen allen skeptischen Kommentaren, die gegenüber diesen methodischen Kategorien vorgebracht wurden, gibt es interessante Situationen, in denen sie meiner Ansicht nach hilfreich sind und sich produktiv überlagern können. Ich möchte das an einem Beispiel erläutern, das gewissermaßen in einer Lücke von Wußings großer Studie zum Gruppenbegriff angesiedelt ist, der Kristallographie des frühen 19. Jahrhunderts. Die erste Übertragung des expliziten, aber noch nicht formalisierten Gruppenbegriffs aus der Auflösungstheorie algebraischer Gleichungen in die Geometrie erfolgte durch eine Arbeit Camille Jordans im Jahre 1869, zwei Jahre vor dessen großer Studie über die groupes des substitutions. Jordan verwies darauf, dass man die Studien der Kristallsymmetrien von Bravais aus mathematischer Sicht als eine geometrische Klassifikation räumlicher Bewegungsgruppen auffassen konnte. Einen Hinweis darauf findet man in Wußings Buch (S. 146). Bei genauerem Hinsehen zeigt sich, dass die Kristallographen des frühen 19. Jahrhunderts in ihren Symmetriestudien erstaunlich weitgehende Einsichten in Strukturen gewonnen hatten, die man seit dem ausgehenden 19. Jahrhundert 
durch (diskrete) Gruppenoperationen beschreiben würde (Scholz 1989).

Ein interessantes Beispiel, wenn auch historisch für die Entwicklung der Kristallographie wohl kaum ausschlaggebend, findet sich in den Arbeiten von Justus G. Grassmann, dem Vater des Begründers der Ausdehnungslehre und damit der (später sogenannten) n-dimensionalen reellen Vektorräume, Hermann G. Grassmann (Petsche 2006, Schubring 1996). J. Grassmann entwickelte in den 1820er Jahren die ,geometrische Combinationslehre“, um die Zusammensetzung der Kräftesysteme, die nach Ansicht der dynamistischen Kristallographen des frühen 19. Jahrhunderts den typischen Aufbau der Kristallgestalten hervorrufen sollten, symbolisch zu beschreiben. Deren Konfigurationen konnte er mit dieser Methode bezüglich ihrer Symmetrien sehr markant klassifizieren und die zugehörigen möglichen Kristallgestalten systematisch auswerten (Grassmann 1833). Er verwendete dazu eine bemerkenswerte multiplikativ notierte algebraische Notation der Zusammensetzung ganzzahlig vervielfältigter sogenannter Elementarträger, den geometrischen Repräsentanten der Grundkräfte des unterstellten Kräftesystems. Symmetrien konnte er durch Permutationen und Vorzeichenwechsel der Koeffizienten (Grassmann nannte sie Wiederholungsexponenten) der jeweiligen Grundkräfte ausdrücken. So konnte er sechs der späteren sieben Kristallsysteme charakterisieren, mathematisch in ihrem (hypothetischen) Kräfteaufbau beschreiben und zugehörige Kristallgestalten in systematischer Weise ableiten. Diese von J. Grassmann etablierte kleine epistemische Struktur enthielt einen gedanklichen Keim für die spätere Ausarbeitung der Ausdehnungsgebiete durch seinen Sohn verbunden mit Gruppierungen (umgangssprachlich zu lesen) von Substitutionen von Wiederholungsexponenten. Mit ganz ähnlich gebauten Substitutionssystemen arbeitete etwa zur selben Zeit Évariste Galois bei seinen Studien zur Auflösungstheorie algebraischer Gleichungen und begann dabei, das Wort groupe auch in einem technisch zugespitzten Sinn zu verwenden (Wußing 1965: $11)$.

Wir sehen an diesem Beispiel, wie tief die von Wußing konstatierten drei Wurzeln des Gruppenbegriffs auch von der Ostension her reichen konnten. Dies kann man allerdings nur dann wahrnehmen, wenn der historische Blick begriffliche Strukturen nicht erst dann (an-)erkennt, wenn gewissermaßen das Ei gelegt und begackert wird, das heißt die Struktur ausgepackt und explizit benannt wird. Entgegen der Kritik und sogar Ablehnung gegenüber solchem Einbezug des Impliziten, dem ich in Diskussionen um die 
Methodologie der Mathematikgeschichte immer wieder begegnet bin, sehe ich die Vertiefung des historischen Blicks auf die impliziten Heranreifungsphasen epistemischer Strukturen, exemplarisch ausgeführt an der Entstehung und Ausarbeitung des Gruppenbegriffs, als ein großes Verdienst Wußings an.

Auch das Einbeziehen von Tätigkeitsbereichen über den professionellen Rahmen des Faches Mathematik hinaus kann den historischen Blick auf das, was an Mathematischem gedacht, ausgeformt und genutzt wird, erheblich anreichern. So paradox es erscheinen mag, die Ostension des Impliziten, oder, wenn man diese Wörter nicht mag, das Sich-Zeigen der noch heranreifenden Erkenntnisstrukturen gehört aus meiner Sicht zu dem Besten, worauf uns Wußing in seinen Studien zu den drei Wurzeln des Gruppenbegriffs aufmerksam gemacht hat. Bloch nannte das den Vor-Schein des Real-Möglichen. Es gibt ihn auch in der Wissenschaftsgeschichte.

\section{Literatur}

Corry, Leo, 1996. Modern Algebra and the Rise of Mathematical Structures. Basel: Birkhäuser.

Epple, Moritz, 1999. Die Entstehung der Knotentheorie. Braunschweig: Vieweg.

Grassmann, Justus G., 1833. Combinatorische Entwicklung der Krystallgestalten. Annalen der Physik und Chemie, 106, 1-43. [Ergänzungsheft].

Gray, Jeremy, 2008. Plato's Ghost. The Modernist Transformation of Mathematics. Princeton: University Press.

Mehrtens, Herbert, 1979. Die Entstehung der Verbandstheorie. Hildesheim: Gerstenberg.

Mehrtens, Herbert, 1990. Moderne, Sprache, Mathematik. Eine Geschichte des Streits um die Grundlagen der Disziplin und des Subjekts formaler Systeme. Frankfurt a. M.: Suhrkamp.

Petsche, Hans-Joachim, 2006. Graßmann. Basel: Birkhäuser. [Vita Mathematica, 13].

Purkert, Walter, 1971. Zur Genesis des abstrakten Körperbegriffs. 1. Teil. NTM. Schriftenreihe für Geschichte der Naturwissenschaften, Technik und Medizin, 8, 23-37.

Purkert, Walter, 1972. Die Entwicklung des abstrakten Körperbegriffs. Dissertation, Karl-Marx-Universität Leipzig.

Purkert, Walter, 1973. Zur Genesis des abstrakten Körperbegriffs. 2. Teil. NTM. Schriftenreihe für Geschichte der Naturwissenschaften, Technik und Medizin, 10, 8-20.

Rheinberger, Hans-Jörg, 1997. Toward a History of Epistemic Things: Synthesizing Proteins in a Test Tube. Stanford: University Press.

Schlote, Karl-Heinz, 1983. Zur Geschichte der Algebrentheorie. Peirces „Linear Associative Algebra“. NTM. Schriftenreihe für Geschichte der Naturwissenschaften, Technik und Medizin, 20, 1-20.

Schlote, Karl-Heinz, 1986/87. Hermann Grassmanns Beitrag zur Algebrentheorie. Janus, 72, 225-255. 
Schlote, Karl.Heinz, 1987. Die Entwicklung der Algebrentheorie bis zu ihrer Formulierung als abstrakte algebraische Theorie. Habilitation Karl-MarxUniversität Leipzig.

Scholz, Erhard, 1980. Geschichte des Mannigfaltigkeitsbegriffs von Riemann bis Poincaré. Basel: Birkhäuser.

Scholz, Erhard, 1989. Symmetrie - Gruppe - Dualität. Zur Beziehung zwischen theoretischer Mathematik und Anwendungen in Kristallographie und Baustatik des 19. Jahrhunderts. Basel: Birkhäuser.

Schubring, Gert, 1978. Das genetische Prinzip in der Mathematik-Didaktik. Stuttgart: Klett-Cotta.

Schubring, Gert, Hg., 1996. Hermann Günther Graßmann (1809-1877). Visionary Mathematician, Scientist and Neohumanist Scholar. Papers from a Sesquicentennial Conference. Dordrecht: Kluwer.

Toeplitz, Otto, [1949] 1972. Die Entwicklung der Infinitesimalrechnung. Eine Einleitung in die Infinitesimalrechnung nach der genetischen Methode. Berlin: Springer [Aus dem Nachlass herausgegeben von Gottfried Köthe. Reprint Darmstadt: Wissenschaftliche Buchgesellschaft].

Wußing, Hans, 1967. Zum historischen Verhältnis von Intension und Extension des Begriffes Gruppe im Herausbildungsprozeß des abstrakten Gruppenbegriffs. NTM. Schriftenreihe für Geschichte der Naturwissenschaften, Technik und Medizin, 4, 23-34.

Wußing, Hans, 1969. Die Genesis des abstrakten Gruppenbegriffes. Berlin (Ost): Verlag der Wissenschaften.

Wußing, Hans, 1970. Zur Entwicklungsgeschichte naturwissenschaftlicher Begriffe. NTM. Schriftenreihe für Geschichte der Naturwissenschaften, Technik und Medizin, 7, 15-29.

Wußing, Hans, 1984. Genesis of the Abstract Group Concept. Cambridge, Mass.: MIT Press.

\section{Erhard Scholz}

Bergische Universität Wuppertal,

Fachbereich C, Mathematik,

42097 Wuppertal,

Deutschland

E-Mail: Erhard.Scholz@math.uni-wuppertal.de 